\title{
RBP4 regulates trophoblastic cell proliferation and invasion via the PI3K/AKT signaling pathway
}

\author{
HONGXIA LI, GUANGMING CAO, NAWEI ZHANG, TONG LOU, \\ QIUSHI WANG, ZHENYU ZHANG and CHONGDONG LIU \\ Department of Obstetrics and Gynecology, Beijing Chao-Yang Hospital, \\ Capital Medical University, Beijing 100020, P.R. China
}

Received January 18, 2018; Accepted June 27, 2018

DOI: $10.3892 / \mathrm{mmr} .2018 .9240$

\begin{abstract}
Insufficient trophoblast invasion is associated with preeclampsia (PE) development. Retinol-binding protein 4 (RBP4) is important for regulating cell differentiation, migration and invasion. The aim of the present study was to determine RBP4 expression and function in the human placenta and to examine the underlying mechanisms. In the present study, RBP4 expression was determined in serum samples from 35 pregnant women with $\mathrm{PE}$ and 30 healthy pregnant women using enzyme-linked immunosorbent assays. Cell proliferation was assessed by Cell Counting Kit- 8 assays, and cell invasion was examined with transwell assays. RBP4 concentrations were significantly lower in the PE group when compared with the control group. RBP4 overexpression enhanced HTR8/SVneo cell proliferation and invasion, and the levels of phosphorylated (p-) phosphoinositide 3-kinase (PI3K) and p-protein kinase B (AKT) in HTR8/SVneo cells. RBP4 knockdown significantly inhibited HTR8/SVneo cell proliferation and invasion, and repressed the expression of matrix metalloproteinases. In addition, RBP4 knockdown significantly reduced the levels of $\mathrm{p}-\mathrm{PI} 3 \mathrm{~K}$ and $\mathrm{p}-\mathrm{AKT}$ in HTR8/SVneo cells. Taken together, the results of the present study demonstrated that RBP4 overexpression increased HTR8/SVneo cell proliferation and invasion by suppressing PI3K/AKT signaling and RBP4 knockdown induced the opposite effects.
\end{abstract}

\section{Introduction}

Preeclampsia (PE) is a pregnancy-specific clinical disorder that affects approximately $3-8 \%$ of all pregnant women (1). PE is

Correspondence to: Professor Zhenyu Zhang or Professor Chongdong Liu, Department of Obstetrics and Gynecology, Beijing Chao-Yang Hospital, Capital Medical University, 8 Gongti South Road, Chaoyang, Beijing 100020, P.R. China

E-mail: zhang_zhenyu112@sina.com

E-mail: liu_dong12@sina.com

Key words: retinol-binding protein 4, preeclampsia, trophoblast, placenta, proliferation, invasion, phosphoinositide 3-kinase/protein kinase B defined by new-onset hypertension and proteinuria after 20 weeks of gestation and is a leading cause of maternal and perinatal mortality (2). A successful pregnancy depends on well-regulated trophoblast invasion into the uterine decidua and moderate uterine spiral artery remodeling. To date, the pathophysiology of PE has not been fully elucidated.

Serum retinol-binding protein 4 (RBP4) is a binding partner of retinol, which is delivered into the circulation by the carrier protein transthyretin (TTR) (3); the formation of this complex increases the molecular weight of RBP4, thus preventing its glomerular filtration and subsequent catabolism in the kidneys. Under physiological conditions, the liver is the major source of RBP4, producing approximately $80 \%$ of this protein. However, RBP4 can also be produced in adipose tissues (4). Chen et al (5) showed that RBP4 is not only a carrier of retinol but also acts as a circulating cytokine. Our previous study using surface-enhanced laser desorption ionization time-of-flight mass spectrometry (SELDI-TOF-MS) revealed that RBP4 is downregulated in PE (6). Serum samples were analyzed using a peptide ligand library conjugated to beads and liquid chromatography-mass spectrometry/mass spectrometry; RBP4 concentrations were found to be significantly lower in women with severe PE than in women with a healthy pregnancy. Immunohistochemistry (IHC) demonstrated significantly lower RBP4 expression (brown) in preeclamptic placental tissues than in normal placental tissues (7).

During placental development, trophoblasts with reduced invasive ability fail to deeply invade the myometrium and to appropriately remodel the uterine spiral arteries, resulting in a shallow placental bed and ultimately leading to PE. In our study, we hypothesized that RBP4 participates in the regulation of trophoblastic cell invasion and migration. The aim of the present study was to investigate the effect of RBP4 on the biological behavior of trophoblasts and to explore the potential signaling pathways involved in this process.

\section{Materials and methods}

Patients and clinical samples. The study protocol was approved by the Ethics Committee of Beijing Chao-Yang Hospital (Beijing, China). All women enrolled in the present study were Chinese patients at the Department of Obstetrics and Gynecology in Chao-Yang Hospital, Capital Medical 
University in Beijing, China, and all patients provided written informed consent before inclusion. Thirty-five patients with $\mathrm{PE}$ and thirty healthy pregnant women were recruited for enzyme-linked immunosorbent assay (ELISA) analysis. PE was defined as the onset of hypertension (systolic blood pressure $\geq 140 \mathrm{mmHg}$ and/or diastolic blood pressure $\geq 90 \mathrm{mmHg}$ on at least two occasions, $4 \mathrm{~h}$ to 1 week apart) after 20 weeks of gestation with proteinuria ( $\geq 300 \mathrm{mg}$ in 24-h urine collection or at least one dipstick measurement $\geq 2+$ ). The control patients were pregnant women who underwent cesarean section because of malposition and premature rupture of membranes. None of the participants had any history of hypertension, diabetes, cardiovascular disease, kidney disease, hyperthyroidism, smoking, alcoholism, chemical dependency, intrauterine fetal death, fetal congenital or chromosomal abnormalities or pregnancies conceived by in vitro fertilization. Blood was drawn via venipuncture and collected in a serum-separator tube. Serum was separated by centrifugation at $1,300 \mathrm{x}$ g and $4{ }^{\circ} \mathrm{C}$ for $10 \mathrm{~min}$ within $2 \mathrm{~h}$ of collection and was stored at $-80^{\circ} \mathrm{C}$ until analysis.

ELISA. ELISAs were conducted according to the manufacturer's instructions (Cloud-Clone Corp., Katy, TX, USA). In brief, $100 \mu \mathrm{l}$ of diluted standards was added to each well containing the quality control and the samples, and the plate was incubated on an orbital microplate shaker at room temperature for $1 \mathrm{~h}$. After the wells were washed three times, $100 \mu \mathrm{l}$ of conjugate solution was added, and the plate was then incubated for $1 \mathrm{~h}$ at room temperature while shaking at $300 \mathrm{rpm}$. The plate was washed three times with wash buffer, $100 \mu 1$ of the substrate solution was added to each well, and the plate was incubated for approximately $10 \mathrm{~min}$ to enable the reaction to develop. Absorbance at $450 \mathrm{~nm}$ was measured using an ELISA plate reader.

IHC. RBP4 expression in placenta tissue was assessed using PV-9000 (standard polymer detection system) for immunohistological staining. IHC was performed to detect RBP4 expression and localization in the placenta. Tissue samples were fixed with sodium phosphate buffer containing $10 \%$ formalin, embedded in paraffin and sliced into $5-\mu \mathrm{m}$ continuous sections. The sections were deparaffinized, rehydrated, and incubated with $3 \% \mathrm{H}_{2} \mathrm{O}_{2}$ in methanol for $30 \mathrm{~min}$ to quench endogenous peroxidase activity. After a short rinse, the sections were heated in a $37^{\circ} \mathrm{C}$ water bath for 15-20 min in citrate buffer. Following cooling and rinsing, the sections were incubated with a rabbit polyclonal anti-RBP4 antibody (1:50) for $30 \mathrm{~min}$ at room temperature, overnight at $4^{\circ} \mathrm{C}$, and for $30 \mathrm{~min}$ at room temperature while shaking. Antibody binding was amplified for $10 \mathrm{~min}$ using biotin and horseradish peroxidase-conjugated streptavidin. The slides were counterstained with hematoxylin, dehydrated in gradient ethanol, cleared in dimethylbenzene, and observed under a microscope. Normal controls for IHC were prepared under the same conditions, 5 placental specimens for each group. The immunostained sections were examined using a Leica DMLA light microscope (Leica Microsystems, Wetzlar, Germany) to assess the prevalence of DAB-positive staining and immunostaining localization within the tissues. All sections were assessed on a microscope for positive DAB staining. Trophoblast cells with unequivocal staining of granular cytoplasm were considered positive. RBP4 expression was evaluated via a computer assessment and scored based on the cytoplasmic staining intensity throughout the entire section. Immunohistochemical staining pictures were taken with the same exposure time by microscope (MOTIC BA400) coupled with camera device (MOTICCAM 2306) in the same microscope environment and conditions. The expressions of target proteins were quantified and the average optical density (AOD) was analyzed by Motic Image Advanced v.3.2 software (MOTIC, Xiamen, China).

Cell culture. The human immortalized trophoblast cell line HTR8/SVneo was obtained from ATCC. The cells were transiently transfected to overexpress or knockout RBP4, and unaltered HTR8/SVneo cells served as the control. The cells were cultured in RPMI 1640 medium (HyClone; GE Healthcare Life Sciences, Logan, UT, USA) supplemented with $10 \%$ fetal bovine serum (FBS; Gibco; Thermo Fisher Scientific, Inc., Waltham, MA, USA), $100 \mathrm{U} / \mathrm{ml}$ penicillin, and $100 \mathrm{mg} / \mathrm{ml}$ streptomycin in a $37^{\circ} \mathrm{C}$ humidified incubator $\left(5 \% \mathrm{CO}_{2}\right)$. The cells were subcultured at a 1:3 ratio when they reached $80-90 \%$ confluence (8).

Lentivirus infection. cDNA containing the human RBP4 sequence was cloned into a pLVX-mCMV-ZsGreen-PGKPuro vector using EcoRI and BamHI digestion. A sequencing analysis was conducted to confirm correct insertion of the DNA fragment. 293T cells (Xi Bei Hong Cheng Biological Technology, Co., Beijing, China) were used to generate packaging lentiviruses for transduction. HTR cells were infected with the pLVX-RBP4-mCMV-ZsGreen-PGK-Puro construct. Finally, stable cell lines were screened.

We used pLVX-shRNA2-Puro lentiviral vectors carrying short hairpin RNAs (shRNAs). The shRNA vectors were obtained from Health and Biological Company, and the lentiviruses were obtained via overnight triple co-transfection of 293 T cells. Knockdown efficiency was determined by reverse transcription-polymerase chain reaction (RT-PCR). We found that the best interference effect was achieved using shRNA2-RBP4-2. HTR cells were infected with the pLVXshRNA2-RBP4-Puro construct, followed by puromycin selection to establish stable cell lines.

Western blotting. Total cell protein was extracted from cells in each group. After centrifugation at $12,000 \mathrm{rpm}$ and $4^{\circ} \mathrm{C}$ for $15 \mathrm{~min}$, the protein concentration in the supernatant was determined with a BCA protein assay. Equal amounts of total protein $(30 \mu \mathrm{g})$ were loaded and separated on $10 \%$ sodium dodecyl sulfate-polyacrylamide gel electrophoresis (SDS-PAGE) gels and transferred to nitrocellulose (NC) membranes using standard procedures.

The primary antibodies rabbit polyclonal anti-MMP2 (1:1,000; Abcam, Cambridge, UK), rabbit polyclonal antiMMP9 (1:1,000; Abcam), rabbit polyclonal anti-AKT (1:1,000; Abcam), rabbit polyclonal anti-p-AKT (Thr308, Ser473) (1:1,000; Abcam), mouse polyclonal anti-PI3K (1:1,000; Abcam), rabbit polyclonal anti-p-PI3K (1:1,000; Abcam), rabbit polyclonal anti-Bcl-2 (1:2,000; Abcam), rabbit 
Table I. Subject characteristics for ELISA.

\begin{tabular}{lcrr}
\hline Characteristics & Controls $(\mathrm{n}=30)$ & PE $(\mathrm{n}=35)$ & P-value \\
\hline Age (years) & $33.3 \pm 2.4$ & $33 \pm 3.6$ & 0.328 \\
Gestational age (weeks) & $37 \pm 6.5$ & $34.7 \pm 7.4$ & 0.066 \\
Blood pressure (mmHg) & & & \\
SBP & $119.2 \pm 15.3$ & $166.4 \pm 13.2$ & $<0.0001$ \\
DBP & $75.5 \pm 9.3$ & $96.8 \pm 12.6$ & $<0.0001$ \\
\hline
\end{tabular}

SBP, systolic blood pressure; DBP, diastolic blood pressure; PE, preeclampsia.

polyclonal anti-GAPDH (1:2,000; Abcam), and rabbit polyclonal anti-RBP4 were used. GAPDH served as the internal loading control.

The membranes were probed with primary antibodies diluted in 5\% SBS/PBS-Tween 20 (PBST-20) overnight at $4^{\circ} \mathrm{C}$, rinsed three times with PBST-20 and then incubated with secondary antibodies $(1: 3,000)$ for $2 \mathrm{~h}$ at room temperature. Excess secondary antibody was removed by four washes in PBST-20. The targeted protein bands were visualized and imaged using an ECL western blotting kit (CWBiotech, Beijing, China), and densitometry was performed using ImageJ (v.1.49e; National Institutes of Health, Bethesda, MD, USA).

In vitro invasion assay with Matrigel. Cell invasion was assessed using transwell chambers (24-well inserts; $8-\mu \mathrm{m}$ pore size) precoated with Matrigel $(200 \mu \mathrm{g} / \mathrm{ml}$; BD Biosciences, Franklin Lakes, NJ, USA). Briefly, 24 h after transfection, HTR8-SVneo cells (RBP4 knockdown, RBP4 overexpression, and control) were trypsinized and seeded into the upper chambers $\left(5 \times 10^{5}\right.$ cells/chamber $)$ in serum-free medium, and the lower chambers were filled with $600 \mu$ l of RPMI 1640 medium containing $10 \%$ FBS as a chemoattractant. After incubation of the plates for $48 \mathrm{~h}$, the cells on the upper surface of the membrane were gently removed, and the invasive cells on the lower surface of the membrane were fixed in methanol and stained with crystal violet. The invasive cells in four randomly selected fields of view were imaged using a light microscope and counted.

Cell treatment. RBP4 knockdown, RBP4 overexpression and control HTR8/SVneo cells $(5,000 /$ well) were seeded into 96-well plates. A CCK8 Assay Kit was used to assess cell survival at different time points $(0,24,48,72,96$ and $144 \mathrm{~h})$. Optical density (OD) was measured at $450 \mathrm{~nm}$. These experiments were performed three times.

Statistical analysis. Statistical analysis was performed using SPSS v.20.0 software (IBM Corp., Armonk, IL, USA). The data were tested for normality using unpaired t-tests, one-way analysis of variance with a least significant difference post hoc test and nonparametric tests (median test). Normally distributed data are presented as the mean \pm standard deviation, and non-normally distributed data, as the median (interquartile range). $\mathrm{P}<0.05$ was considered to indicate a statistically significant difference.

\section{Results}

Subject characteristics. Blood samples from thirty-five patients with PE and thirty healthy pregnant women were analyzed via ELISA. The patient characteristics are listed in Table I.

IHC to assess RBP4 expression in the placenta. IHC analysis showed that RBP4 localized to syncytiotrophoblasts (STBs). $\mathrm{RBP} 4$ expression in PE placenta tissue $(\mathrm{n}=5)$ was significantly decreased compared with that in normal controls $(n=5$; Fig. 1$)$. Normal placenta tissue stained for RPB4 at $0.79 \pm 0.05$; PE placenta tissue stained for RPB4 at $0.39 \pm 0.02$.

ELISA. Serum RBP4 expression was compared between patients with PE and healthy pregnant women using ELISA (PE median, $129.3 \mathrm{ng} / \mathrm{ml}$ vs. control median, $166.7 \mathrm{ng} /$ $\mathrm{ml} ; \mathrm{P}=0.046 ;$ standard curve line $\mathrm{y}=0.0176 \mathrm{x}+0.1724$ $\left(\mathrm{R}^{2}=0.9527\right)$; Fig. 2).

RBP4 regulates HTR8/SVneo cell proliferation in vitro. We investigated whether RBP4 affects the proliferation of trophoblasts. We found that overexpression of RBP4 increased cell proliferation after 120 and $144 \mathrm{~h}$, whereas siRNA-mediated knockdown of RBP4 significantly decreased HTR8/SVneo cell proliferation at 24, 48 and $72 \mathrm{~h}$ (Fig. 3). Two bands for RBP4 should be present in the western blot of the RBP4 overexpression group. However, RBP4 was cloned into the vector without including vsgreen in the frame, resulting in overexpression of RBP4 without any tags. Therefore, only one band was observed when blotting with the anti-RBP4 antibody. Western blot analysis of RBP4 to confirm overexpression and knock down was successful.

In vitro invasion assay. Cell invasion assays were employed to investigate the effect of RBP4 expression on HTR8/SVneo cell invasion (Fig. 4). RBP4 overexpression promoted the invasion of HTR8/SVneo cells (A group), and RBP4 knockdown decreased the invasion of HTR8/SVneo cells (B group).

Effect of RBP4 on MMP2, MMP9 and Bcl-2 expression. MMP2, MMP9, and Bcl-2 are associated with invasion and apoptosis. Therefore, we measured the protein levels of MMP2, MMP9 and Bcl-2 via western blotting. Overexpression of RBP4 significantly increased MMP2, MMP9 and Bcl-2 expression in HTR8/SVneo cells (B group), and knockdown of RBP4 decreased MMP2, MMP9 and Bcl-2 expression in 


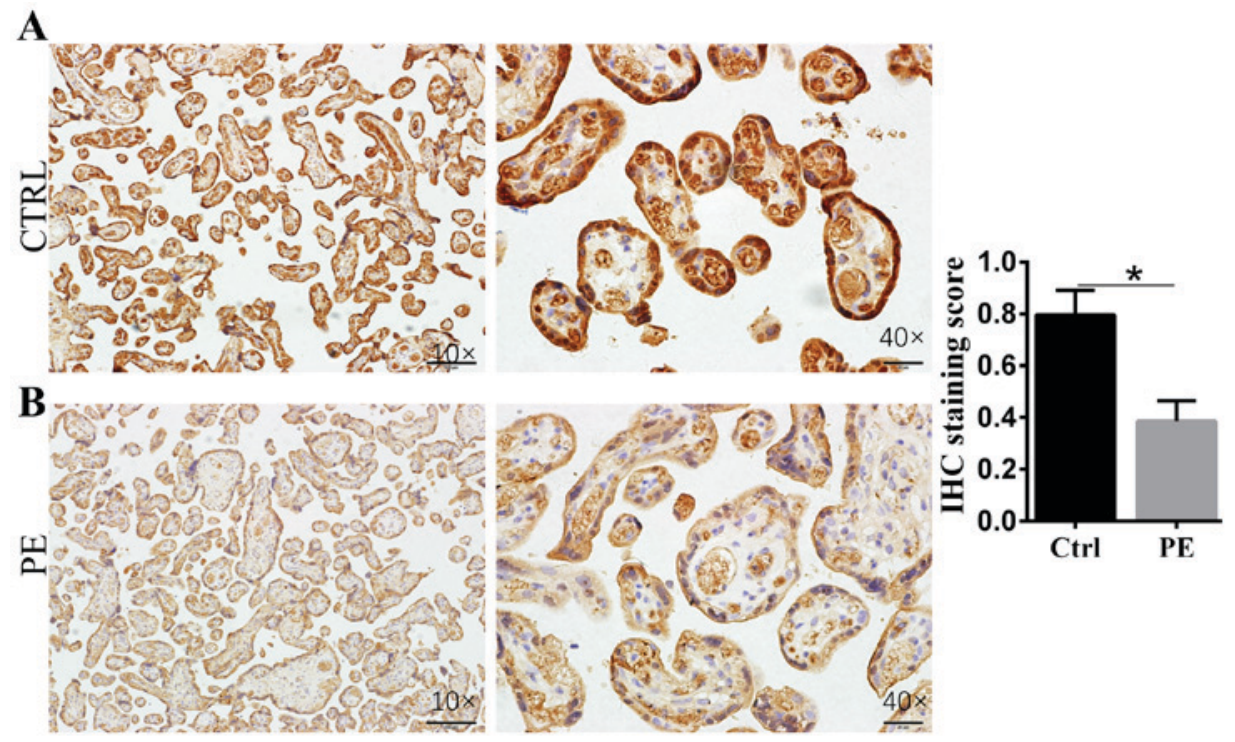

Figure 1. IHC analysis of RBP4 expression in the placenta. Example of immunostaining: Positive antibody staining is brown. (A) RBP4 immunostaining of the CTRL group; and (B) RBP4 immunostaining of the PE group (magnification, $\mathrm{x} 10$ and $\mathrm{x} 40$, as indicated). $\mathrm{P}<0.05$, as indicated. IHC, immunohistochemistry; RBP4, Retinol-binding protein 4; CTRL, control; PE, preeclampsia.

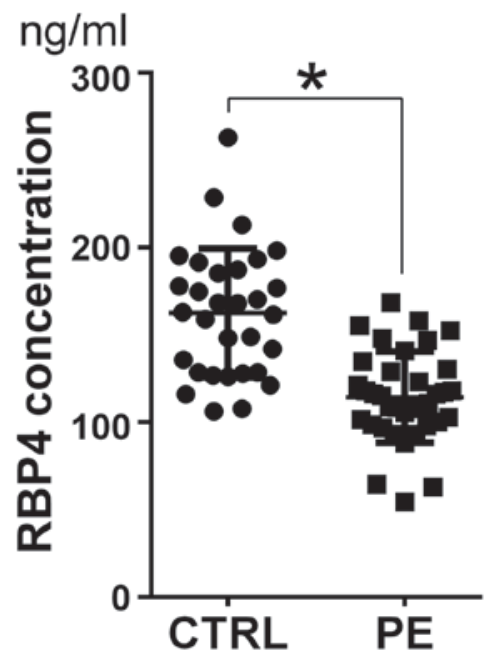

Figure 2. Serum RBP4 expression based on ELISA. Serum RBP4 concentrations were significantly lower in the PE group when compared with the control group. ${ }^{*} \mathrm{P}<0.05$, as indicated. RBP4, Retinol-binding protein 4 ; CTRL, control; PE, preeclampsia.

HTR8/SVneo cells (A group). Each experiment was repeated three times (Fig. 5).

Effect of RBP4 on the PI3K/AKT signaling pathway in HTR8/ SVneo cells. To elucidate the mechanisms by which RBP4 promotes trophoblast invasion, we evaluated PI3K/AKT signaling in trophoblast cells. We examined PI3K, AKT, p-PI3K and $\mathrm{p}$-AKT expression via western blotting. Each experiment was repeated three times (Fig. 6). p-AKT (Ser473) and p-PI3K were significantly upregulated in the RBP4 overexpression group compared with the normal control group, and there were no differences in AKT and PI3K levels between the groups (B group). p-AKT (Ser473), p-AKT (Thr308) and p-PI3K were significantly downregulated in the RBP4 knockdown group compared with the normal control group (A group).

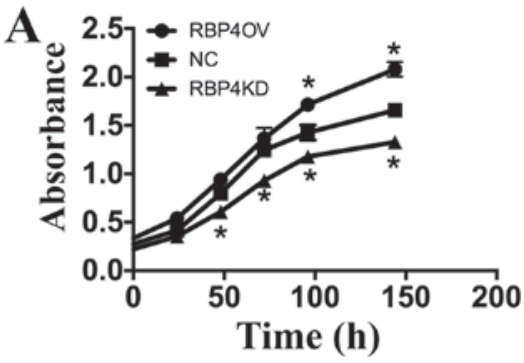

B

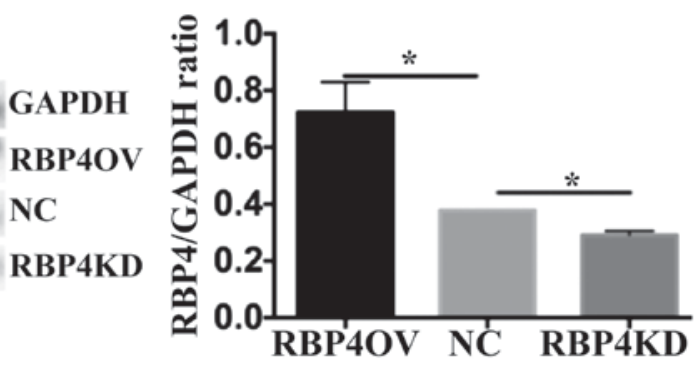

Figure 3. RBP4 regulates HTR8/SVneo cell proliferation in vitro. (A) RBP4 overexpression increased cell proliferation, and RBP4 knockdown inhibited HTR8/SVneo cell proliferation. The proliferation of control cells, RBP4overexpressing cells and RBP4 knockdown cells at $0,24,48,72,120$ and $144 \mathrm{~h}$ was determined by Cell Counting Kit- 8 assays. The absorbance of viable cells was determined at $450 \mathrm{~nm}$ (optical density 450). Proliferation of RBP4-overexpressing cells was significantly higher than that of the control cells at 96 and $144 \mathrm{~h}$. Proliferation of RBP4-knockdown cells was significantly lower than that of the control cells at 48, 72, 96 and $144 \mathrm{~h}$. ${ }^{*} \mathrm{P}<0.05$ vs. NC. (B) Western blot analysis of RBP4 protein expression to confirm that overexpression and knockdown were successful. ${ }^{*} \mathrm{P}<0.05$, as indicated. All quantitative data are presented as the mean \pm standard deviation of three independent experiments. RBP4, Retinol-binding protein 4; NC, normal control; RBP4OV, RBP4 overexpression group; RBP4KD, RBP4 knockdown group.

\section{Discussion}

PE is generally defined as new-onset hypertension accompanied by proteinuria or complications in multiple organs at or after the 20th week of gestation $(9,10)$. Although extensive 
A CTRL

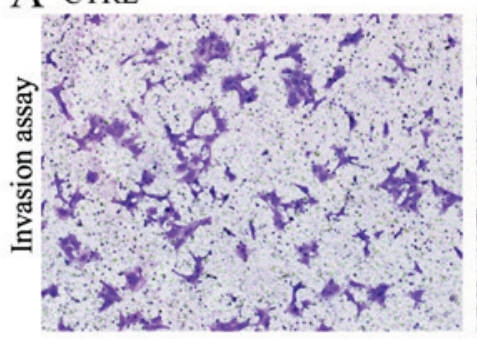

B CTRL

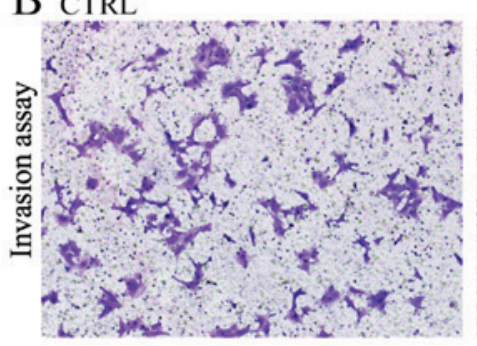

RBP4 overexpress

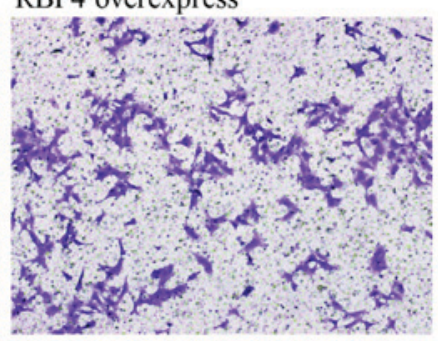

RBP4 knockdown

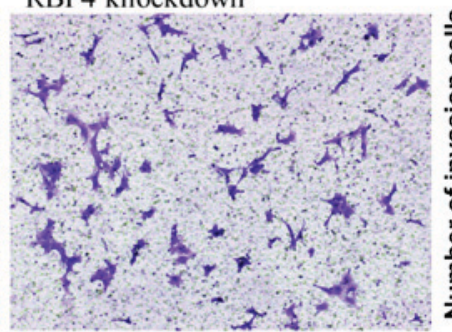

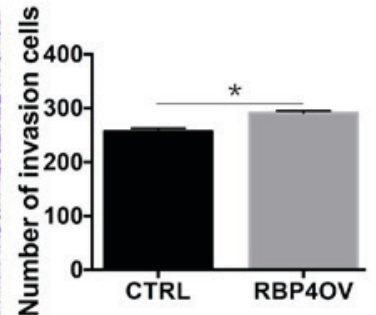

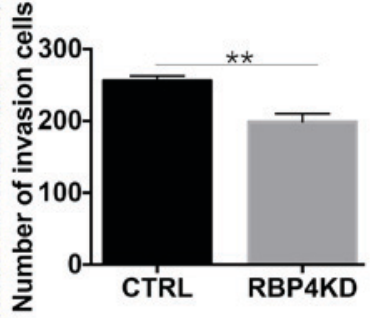

Figure 4. In vitro invasion assay: RBP4 reduces cell invasion. Cell invasion across Matrigel was determined by transwell assays. Images of representative invasive cells are shown on the left (magnification, $\mathrm{x} 10$ ), and quantification analysis is presented on the right. (A) There was significantly increased trophoblast cell invasion in the RBP4 overexpression group. (B) Compared with the control group, cells with RBP4 knockdown displayed significantly reduced invasion ability. All quantitative data are presented as the mean \pm standard deviation of three independent experiments. ${ }^{*} \mathrm{P}<0.05$ and ${ }^{* * *} \mathrm{P}<0.01$, as indicated. $\mathrm{RBP} 4$, Retinol-binding protein 4; CTRL, control; RBP4OV, RBP4 overexpression group; RBP4KD, RBP4 knockdown group.
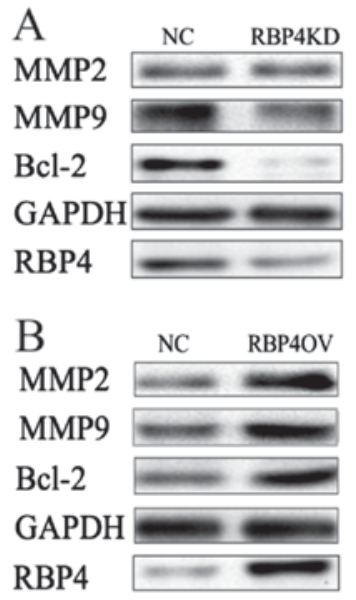
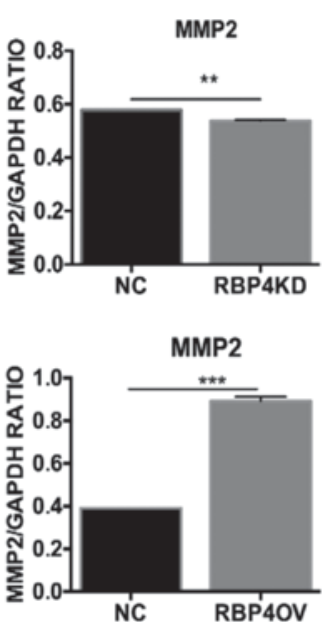
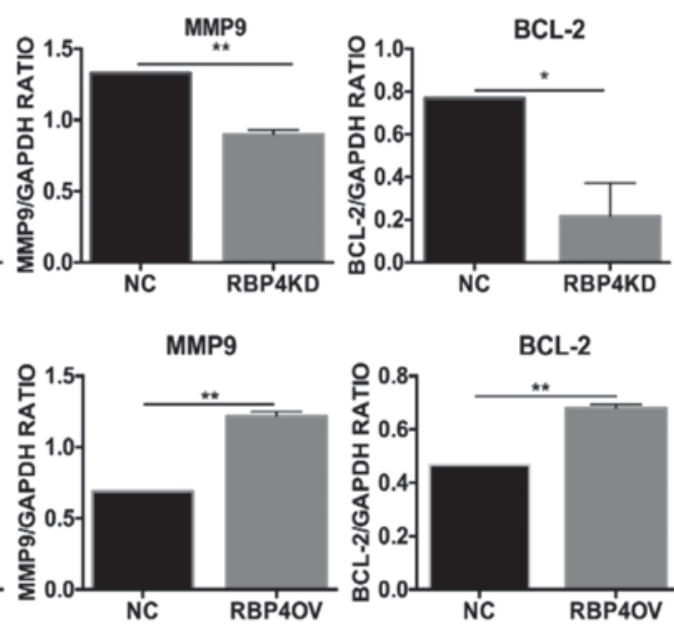

Figure 5. Effect of RBP4 on MMP2, MMP9 and Bcl-2 expression. Effects of RBP4 knockdown and overexpression on the expression of proteins associated with invasion. (A) MMP2, MMP9 and Bcl-2 protein expression was downregulated in the RBP4KD group when compared with the NC group. (B) MMP2, MMP9 and Bcl-2 expression was upregulated in the RBP4OV group when compared with the NC group. ${ }^{*} \mathrm{P}<0.05,{ }^{* *} \mathrm{P}<0.01$ and ${ }^{* * * *} \mathrm{P}<0.001$, as indicated. RBP4, Retinol-binding protein 4; NC, normal control; RBP4OV, RBP4 overexpression group; RBP4KD, RBP4 knockdown group; MMP, matrix metalloproteinase; Bcl-2, B-cell lymphoma 2.

studies have demonstrated that immunologic, antigenic, genetic, metabolic, and environmental factors are associated with PE (11), the pathophysiology of PE has not yet been fully elucidated. Moreover, there are no reliable biomarkers for predicting PE during early pregnancy.

Maintenance of a normal pregnancy depends on placental development and function. Moderate invasion of human extravillous trophoblast cells into the maternal decidual stroma and spiral arteries is essential for establishing a successful pregnancy. Similar to tumor cells, trophoblasts have invasive ability; however, unlike tumor invasion, trophoblast invasion is a strictly controlled physiological event. Abnormal trophoblast differentiation is associated with a variety of pregnancy-associated diseases. Insufficient trophoblast invasion and proliferation are correlated with PE development $(12,13)$.

Recent studies have suggested that RBP4, an integral member of a trimer with retinol and TTR that is transported to specific tissues and organs in the body, may be linked to insulin resistance, type 2 diabetes mellitus, renal dysfunction, atherosclerosis and hypertension in women (14). RBP4 is a $21-\mathrm{kDa}$ protein and a specific carrier of retinol (vitamin A) from the liver to peripheral tissues (15). In the present study, IHC analysis showed that RBP4 is localized in STBs, and RBP4 expression in PE placenta tissue was significantly decreased compared with that in normal controls. In the 

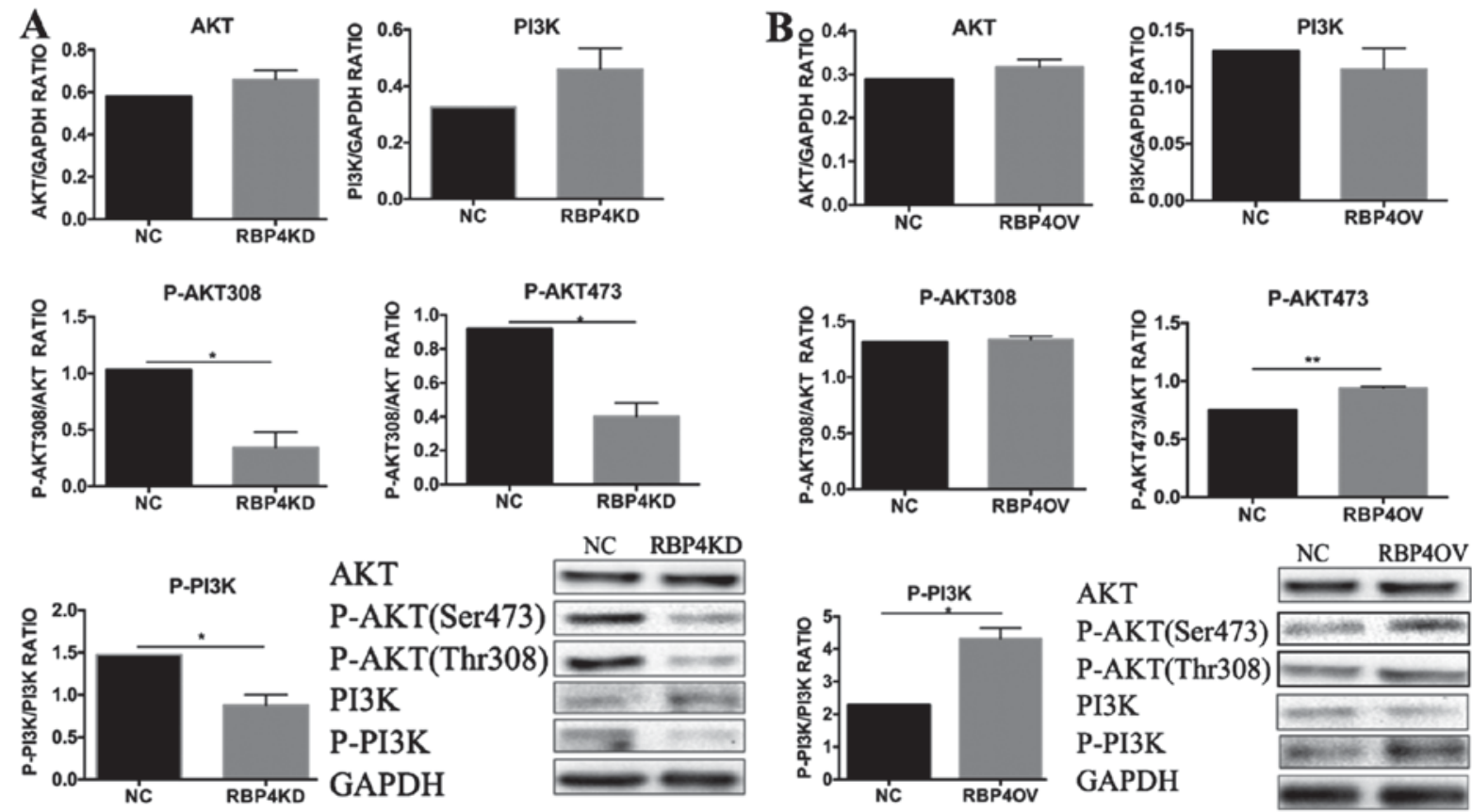

Figure 6. Effect of RBP4KD and RBP4OV on the PI3K/AKT signaling pathway. (A) p-AKT (Ser473), p-AKT (Thr308) and p-PI3K were significantly downregulated in the RBP4KD group when compared with the NC group. (B) p-AKT (Ser473) and p-PI3K were significantly upregulated in the RBP4OV group when compared with the NC group. There were no differences in AKT and PI3K levels between the groups. " $\mathrm{P}<0.05$ and ${ }^{* *} \mathrm{P}<0.01$, as indicated. RBP4, Retinolbinding protein 4; NC, normal control; RBP4OV, RBP4 overexpression group; RBP4KD, RBP4 knockdown group; p-, phosphorylated; AKT, protein kinase B; PI3K, phosphoinositide 3-kinase.

present study, serum RBP4 concentration as determined by ELISA was found to be significantly lower in patients with PE than in healthy pregnant women, consistent with the results of our previous study (7).

The trophoblast invasion process requires degradation and remodeling of the decidual extracellular matrix. Because the MMP family is involved in breakdown of the extracellular matrix, to gain a greater understanding of the mechanisms underlying RBP4-regulated cell invasion, we examined the expression of MMP2 and MMP9, which were previously found to increase the invasiveness of trophoblasts (16-18). We found that RBP4 knockdown decreased cell invasion, and RBP4 overexpression increased the invasion of HTR8/ SVneo cells. Moreover, the production of MMP2 and MMP9 was decreased in RBP4-knockdown HTR8/SVneo cells and dramatically increased in RBP4-overexpressing cells. These results indicated that MMP2 and MMP9 likely contribute to RBP4-induced trophoblast invasion. Thus, it is worthwhile to ascertain whether RBP4 acts directly on MMP2 and MMP9 promoters or functions indirectly through some other factor.

The PI3K/AKT signaling pathway plays important roles in cell growth, proliferation, migration and invasion (19). We found that RBP4 overexpression did not increase HTR8/SVneo cell proliferation at 24,48 or $72 \mathrm{~h}$, and thus, we cultivated the cells for longer periods of time. After 96 and $144 \mathrm{~h}(20,21)$, we found that the overexpression of RBP4 increased cell proliferation, whereas siRNA-mediated RBP4 knockdown significantly decreased HTR8/SVneo cell proliferation at 24, 48, 72, 96 and $144 \mathrm{~h}$. Activation of PI3K/AKT signaling inhibits apoptosis (22) through Bcl-2 (23), an anti-apoptotic protein that is downstream of the PI3K/AKT pathway. Bcl-2 is associated with or may be necessary for cell growth and survival. In our study, Bcl-2 was increased in RBP4-overexpressing cells and decreased in RBP4-knockdown cells. RBP4 is known to activate PI3K/AKT signaling in HTR8/SVneo cells. We found that RBP4 knockdown decreased AKT (Ser473 and Thr308) and PI3K phosphorylation, although the total protein expression of AKT and PI3K remained constant. Therefore, blockade of the PI3K/AKT pathway may be one reason for the cell growth inhibition after RBP4 knockdown.

In summary, our data show that RBP4 is involved in the proliferation and invasion of trophoblastic cells. Additionally, RBP4 can activate the PI3K/AKT signaling pathway and upregulate MMP2 and MMP9 expression. Based on the results of the current study, we hypothesize that RBP4 regulates trophoblastic cell proliferation and invasion by activating the PI3K/AKT pathway. Decreased RBP4 expression in the placenta may contribute to PE development by decreasing the invasive ability of trophoblasts.

Therefore, placental trophoblast cell differentiation indeed involves an EMT-like process, which will be the focus for our follow-up research. The present study is the first to detect the effect of RBP4 on the invasion ability of trophoblast cells and changes in MMP-related molecules, including Snail, Slug, Vimentin, and E-Cadherin, which are directly related to cell invasion ability. We plan to conduct additional studies in the future to further elucidate these mechanisms.

\section{Acknowledgements}

The authors would like to thank the Experimental Center of Capital Medical University, Beijing Chao-Yang Hospital (Beijing, China) for their assistance and cooperation during the present study. 


\section{Funding}

The present study was supported by grants from the National Natural Science Foundation of China (grant no. 81571455) and Sino-RUS Cooperation Funds (grant no. 2015DFR31070).

\section{Availability of data and materials}

The datasets used and/or analyzed during the present study are available from the corresponding author on reasonable request.

\section{Authors' contributions}

ZZ and QW conceived and designed the experiments. HL and TL performed the experiments. ZZ and GC analyzed the data. ZZ and CL interpreted the data, and HL wrote the first draft of the manuscript. ZZ and CL gave final approval of the results and conclusions made in the manuscript. NZ analyzed the results and developed the structure and arguments for the paper. HL, CL and ZZ critically revised the paper for important intellectual content. All authors read and approved the final manuscript.

\section{Ethics approval and consent to participate}

The research protocol was conducted in accordance with the guidelines of the World Medical Association's Declaration of Helsinki and was performed following approval from the Medical Ethics Committee (11-S-59) of Beijing Chao-Yang Hospital, Capital Medical University. All of the women enrolled in the present study provided written informed consent prior to inclusion to the study.

\section{Patient consent for publication}

All of the women enrolled in the present study provided written informed consent prior to inclusion to the study.

\section{Competing interests}

The authors declare that they have no competing interests.

\section{References}

1. Anderson UD, Olsson MG, Kristensen KH, Åkerström B and Hansson SR: Review: Biochemical markers to predict preeclampsia. Placenta 33 (Suppl): S42-S47, 2012.

2. Noris M, Perico N and Remuzzi G: Mechanisms of disease: Pre-eclampsia. Nat Clin Pract Nephrol 1: 98-114; quiz 120, 2005

3. Naylor HM and Newcomer ME: The structure of human retinolbinding protein (RBP) with its carrier protein transthyretin reveals an interaction with the carboxy terminus of RBP. Biochemistry 38: 2647-2653, 1999.

4. Goodman DS: Plasma retinol-binding protein. Ann N Y Acad Sci 348: 378-390, 1980
5. Chen $\mathrm{CH}$, Hsieh TJ, Lin KD, Lin HY, Lee MY, Hung WW, Hsiao PJ and Shin SJ: Increased unbound retinol-binding protein 4 concentration induces apoptosis through receptor-mediated signaling. J Biol Chem 287: 9694-9707, 2012.

6. Liu C, Zhang N, Yu H, Chen Y, Liang Y, Deng H and Zhang Z: Proteomic analysis of human serum for finding pathogenic factors and potential biomarkers in preeclampsia. Placenta 32 : 168-174, 2011.

7. Lu Q, Liu C, Liu Y, Zhang N, Deng H and Zhang Z: Serum markers of pre-eclampsia identified on proteomics. J Obstet Gynaecol Res 42: 1111-1118, 2016.

8. Ding J, Huang F, Wu G, Han T, Xu F, Weng D, Wu C, Zhang X, Yao Y and Zhu X: MiR-519d-3p suppresses invasion and migration of trophoblast cells via targeting MMP-2. PLoS One 10: e0120321, 2015.

9. Sibai B, Dekker G and Kupferminc M: Pre-eclampsia. Lancet 365: 785-799, 2005.

10. Steegers EA, von Dadelszen P, Duvekot JJ and Pijnenborg R: Pre-eclampsia. Lancet 376: 631-644, 2010.

11. Redman CW and Sargent IL: Latest advances in understanding preeclampsia. Science 308: 1592-1594, 2005.

12. Ball E, Bulmer JN, Ayis S, Lyall F and Robson SC: Late sporadic miscarriage is associated with abnormalities in spiral artery transformation and trophoblast invasion. J Pathol 208: 535-542, 2006.

13. Goldman-Wohl D and Yagel S: Regulation of trophoblast invasion: From normal implantation to pre-eclampsia. Mol Cell Endocrinol 187: 233-238, 2002.

14. Solini A, Santini E, Madec S, Rossi C and Muscelli E: Retinol-binding protein- 4 in women with untreated essential hypertension. Am J Hypertens 22: 1001-1006, 2009.

15. Hendler I, Blackwell SC, Mehta SH, Whitty JE, Russell E, Sorokin Y and Cotton DB: The levels of leptin, adiponectin, and resistin in normal weight, overweight, and obese pregnant women with and without preeclampsia. Am J Obstet Gynecol 193: 979-983, 2005.

16. Chen JZ, Wong MH, Brennecke SP and Keogh RJ: The effects of human chorionic gonadotrophin, progesterone and oestradiol on trophoblast function. Mol Cell Endocrinol 342: 73-80, 2011.

17. Jovanović M, Stefanoska I, Radojcić L and Vićovac L: Interleukin-8 (CXCL8) stimulates trophoblast cell migration and invasion by increasing levels of matrix metalloproteinase (MMP) 2 and MMP9 and integrins alpha5 and beta1. Reproduction 139: 789-798, 2010.

18. Karthikeyan VJ, Lane DA, Beevers DG, Lip GY and Blann AD: Matrix metalloproteinases and their tissue inhibitors in hypertension-related pregnancy complications. J Hum Hypertens 27: 72-78, 2013.

19. Duan C, Bauchat JR and Hsieh T: Phosphatidylinositol 3-kinase is required for insulin-like growth factor-I-induced vascular smooth muscle cell proliferation and migration. Circ Res 86: $15-23,2000$.

20. Zhang R, Leng H, Huang J, Du Y, Wang Y, Zang W, Chen X and Zhao G: miR-337 regulates the proliferation and invasion in pancreatic ductal adenocarcinoma by targeting HOXB7. Diagn Pathol 9: 171, 2014.

21. Yan C, Zhu Y, Zhang X, Chen X, Zheng W and Yang J: Downregulated aquaporin 5 inhibits proliferation and migration of human epithelial ovarian cancer 3AO cells. J Ovarian Res 7: 78, 2014.

22. Downward J: Mechanisms and consequences of activation of protein kinase B/Akt. Curr Opin Cell Biol 10: 262-267, 1998.

23. Kemi OJ, Ceci M, Wisloff U, Grimaldi S, Gallo P, Smith GL, Condorelli G and Ellingsen O: Activation or inactivation of cardiac Akt/mTOR signaling diverges physiological from pathological hypertrophy. J Cell Physiol 214: 316-321, 2008.

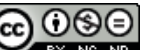

This work is licensed under a Creative Commons Attribution-NonCommercial-NoDerivatives 4.0 International (CC BY-NC-ND 4.0) License. 